\title{
Emerging properties of malaria transmission and persistence in urban Accra, Ghana: Evidence from a participatory system approach
}

Merveille Koissi Koissi Savi ( $\square$ koissi.savi@uni-bonn.de)

Rheinische Friedrich-Wilhelms-Universitat Bonn https://orcid.org/0000-0002-7691-0495

Daniel Callo-Concha

Universitat Koblenz-Landau

Henri E.Z. Tonnang

International Centre for Insect Physiology and Ecology

Christian Borgemeister

Rheinische Friedrich-Wilhelms-Universitat Bonn

\section{Research Article}

Keywords: Network analysis, causal loop diagram, emergence, urban, complex system.

Posted Date: March 29th, 2021

DOI: https://doi.org/10.21203/rs.3.rs-368653/v1

License: (9) This work is licensed under a Creative Commons Attribution 4.0 International License.

Read Full License

Version of Record: A version of this preprint was published at Malaria Journal on July 19th, 2021. See the published version at https://doi.org/10.1186/s12936-021-03851-7. 
Title: Emerging properties of malaria transmission and persistence in urban Accra, Ghana:

Evidence from a participatory system approach

Authors: Merveille Koissi Savi ${ }^{1,}$, Daniel Callo-Concha ${ }^{1,2}$, Henri E.Z. Tonnang ${ }^{3}$ \& Christian Borgemeister $^{1}$

\section{Affiliations:}

${ }^{1}$ University of Bonn, Center for Development Research (ZEF), 53113 Bonn, Germany

${ }^{2}$ University of Koblenz-Landau, Institute for Environmental Sciences (iES), 76829 Landau, Germany

${ }^{3}$ International Centre for Insect Physiology and Ecology (icipe), Nairobi, Kenya

*Corresponding author: E-mail: koissi.savi@ uni-bonn.de 


\begin{abstract}
Several studies that aim to enhance the understanding of malaria transmission and persistence in urban settings failed to address its underlining complexity. We aim at doing that by applying a qualitative and participatory-based system analysis and mapping to elicit the system's emergent properties. In two experts' workshops, we sketched and refined the system, which was represented through a causal loop diagram, where the identification of leverage points was done using network analysis. We found 45 determinants interplaying through 56 linkages, and identified three subsystems: urbanization-related transmission, infection-prone behavior and healthcare efficiency, and Plasmodium resistance. Apart from the number of breeding sites and malaria positive cases, other determinants such as drug prescription and the awareness of householders were identified by the network analysis as leverage points and emergent properties of the system of transmission and persistence of malaria. Based on our findings, we suggest that ongoing efforts to control malaria, such as the use of insecticide-treated bed nets and larvicide applications should continue, and include new ones focusing on the public awareness and malaria literacy of city dwellers. We found that our participatory approach strengthened the legitimacy of the recommendations and the co-learning of participants.
\end{abstract}

Keywords: Network analysis, causal loop diagram, emergence, urban, complex system. 


\section{Background}

Malaria is still the number one deadliest infectious disease, responsible for more than 380,000 deaths in 2018 only [1]. In endemic areas of sub-Saharan Africa (SSA), the expenses for its control and prevention reach up to $40 \%$ of all public health expenditures [2], and its effects were estimated to have reduced the GDP by $9 \%$ in 2010 in affected countries in SSA [3]. Due to the collaborative efforts of governments and development partners, malaria mortality has been reduced by $66 \%$ from 2007 to 2017 [4], but the challenge is yet far from being solved. Africa's population is expected to triple by 2050 [5], with major growth occurring in urban areas. For example, the population of major Ghanaian cities has grown by 3.5\% per annum from 1984 to 2010 [6]. As urbanization brings along challenges, such as traffic congestion, slumming, and pollution, little priority is given to public health-related issues [7]. For example, in Accra, the capital of Ghana, city planning issues often overshadow the health-related ones, with the local government investing less than 50USD per person per year on health [8]. Moreover, the poorest communities experience the greatest harm [8], like Accra's head porters, especially challenged by the nature of their work and often not able to afford the national insurance scheme [9]. A pressing public health issue is the prevalence of vector-borne diseases, like malaria [10,11]. Its predominant vector Anopheles gambiae, whose customary habitat for reproduction used to be rural, clean, and shallow water ponds surrounded by grassy fields, has now adapted to the urban conditions and prospers in polluted waters, such as clogged gutters or puddles, characteristic of poor urban housing $[10,12]$.

This example evidences the complex and adaptive character of the malaria transmission system, where the humans, vectors, the environment, and parasites interact in an iterative and nonlinear manner [13]. Earlier modeling approaches rarely considered such complexity, and instead conceived transmission causal and unilaterally, which contributed to the development of policies 
favoring the promotion of single-intervention programs, like the free provision of malaria drugs [14-16].

A complex system is one where its components, apparently disconnected and performing their roles after their interests, align together to perform more sophisticated functions [17]. This tends to be the case for most social and ecological phenomena, as they do not occur in isolation but intermingled [18]. The unraveling of complex systems is operationalized through approaches, methods, and tools that instead of assessing the determinants individually, focus on the interactions among them, and the overall function of the system [19]. In that regard, the involvement of local stakeholders appears key, as it reduces the bias of researchers and increases the legitimacy of the outcomes [20].

We have applied such approaches in this study and visualized the interactions among the determinants of malaria transmission in urban conditions using a Causal Loop Diagram (CLD) and displayed emergent properties of the system via Network Analysis (NA).

CLD can support the visualization of the interplay among determinants and assist in the identification of causal relationships among them [21]. Moreover, to better understand the complexity in malaria transmission, CLD can also help to devise improved strategies for more effective control of the disease in cities (and beyond) by scouting underplayed channels [13]. NA is based on the principles of network theory, where a system is considered a web of edges (interactions) that connect the nodes (determinants) [22]. The examination of the network properties (e.g. density) and its topology, e.g. centrality, indicate the nature of the information flow and reveal the most sensitive determinants and their roles [23,24]. Furthermore, by applying graph theory, NA offers powerful visualizations of the analyzed phenomena [22,25]. A successful application of NA can display some emerging properties of complex systems, such as 
McGlashan [24] did in identifying the leverage points, i.e., where one can intervene to alter the system of childhood obesity in Australia.

We hypothesized that by combining participatory CLD and NA we would be able to better accomplish the aims of this study, that are (i) to understand the interplay between determinants of the system of transmission and persistence of malaria in urban settings, and subsequently (ii) to identify its emerging properties (i.e., properties of the network and leverage points of the system and derive potential interventions on the system).

\section{Methods}

\section{Study area}

Accra, the capital city of Ghana, is located on the coast of the West African Golf of Guinea. Its climate is tropical alternating wet and dry phases, mainly due to the cyclical harmattan winds. The average annual rainfall is $730 \mathrm{~mm}$ and bimodally distributed, the temperature average reaches $26.6^{\circ} \mathrm{C}$, and the relative humidity rounds $81 \%$, with little variations along the year [26,27]. Accra's current population is 2,3 million, to a great extent composed of migrants from successive waves of rural-urban migration across the last 50 years. Housing is uneven in infrastructure quality and service provision, but standards are generally low. The worst affected areas are old central neighborhoods, where slums abound, and peripheral settlements, where new developments happen [28].

Although malaria is traditionally considered a predominantly country-side disease, recent evidence showed that mortality and morbidity in SSA's cities are high [26]. For example, in Accra, slums and poorly-managed urban areas such as James-Town and Korle-Dudor districts recorded the highest malaria indices of morbidity and mortality [29]. 


\section{Identification of key experts and Causal Loop Diagram elicitation}

Initially, we held an informal meeting with district assembly members of James-Town and Korle-Dudor districts and other members of the communities, to identify the key institutions and experts working on the prevention and treatment of malaria. The list of institutions and experts was consolidated to include twelve representatives from the Ghana National Malaria Program, Malaria Initiative/ USAID, World Health Organization, Ghana Health Service, Plant Protection and Regulatory Services/ Ministry of Food and Agriculture, Noguchi Memorial Institute for Medical Research, several NGOs, and local healthcare facilities.

The experts met in two recorded workshops, which were facilitated by a modeling team (modeler, facilitator, and wall-builder) following Hovmand's guidelines [30].

In the first workshop, we refined the problem, defined the variables of the model, and drew an initial CLD. A CLD aims to show the interplay between components of a complex system, eliciting the feedback loops, and facilitate the understanding of a given problem [31,32]. For that, we set the background by presenting the outcomes of precedent informal interviews; then, together with the experts, the boundaries of the malaria-related transmission and persistence CLD were defined.

Secondly, experts and the modeling team identified a list of determinants explaining the transmission and persistence of malaria and elicited a cause-effect map from the interactions between determinants. A cause in the CLD represents a determinant from which the arrow emerges, and the effect is a determinant that receives the arrow. The positive or negative sign of the arrow explains the type of association, i.e., a cause A implying an effect B showing a positive sign should be read: The increase in A implies an increase in B. Inversely, A implying B with a negative sign should be read: An increase in A causes a decrease in B (Fig. 1). Subsequently, 
determinants that were not relevant were excluded, e.g., indoor residual spray, which is not used in Accra

During the second workshop, together with experts, we proceeded to the validation and refinement of the CLD. In consequence, new determinants were added whereas others were merged into more inclusive ones, and some determinants judged non-relevant were removed, which led to changes in the causal linkages. The model obtained (Suppl. Fig. 1) was further refined by the modeling team, which focused on the identification of feedback loops in the CLD (Suppl. Table1).

\section{Network analysis}

The emerging properties of the system represented by the CLD were displayed by the properties of the network and its most central determinants. The most central determinants stand for the leverage points for transmission and persistence of malaria in Accra. These points can enhance the control of malaria when they are adjusted according to the properties displayed by the system.

The CLD represents an unweighted directed network, where the connectivity is represented by the adjacency non-symmetric and unweighted matrix $A_{i j}$ (Eq. 1) [24]

$$
A_{i j}=\left\{\begin{array}{cc}
1 & \text { if i causally influences j } \\
0 & \text { otherwise }
\end{array}\right.
$$

The properties of the network were estimated through the computation of the density, the average path length, and the modularity of the CLD network. The functional importance of the determinants was captured via the calculation of six measures of centrality, i.e., degree $(K)$, indegree $\left(K^{\text {in }}\right)$, out-degree $\left(K^{\text {out }}\right)$, PageRank $(x)$, closeness $(C)$, and betweenness $(B)$. The degree centrality $(K)$ assesses the determinants' connectivity. With respect to the adjacency matrix, the degree $k_{i}$ can be calculated for a network containing $n$ nodes: 


$$
k_{i}=\sum_{j=1}^{n} A_{i j}
$$

Since the causal diagram is assimilated to a directed network, Eq. 2 can be re-written as

$$
k_{i}^{\text {in }}=\sum_{j=1}^{n} A_{i j}, \quad k_{i}^{\text {out }}=\sum_{i=1}^{n} A_{i j}
$$

where $k_{i}{ }^{i n}$ and $k_{i}^{\text {out }}$ stand for in-degree and out-degree centrality if there is connectivity from $j$ to $i$, which indicates the direction of the connection between determinants, the former as a recipient (effect), and the latter as emitter (cause).

PageRank eigenvector centrality $x_{i}$ value estimates the influence of certain determinants on the whole network $[33,34]$ and is given by

$$
x_{i}=0.85 \times \sum_{i} A_{i j} \frac{b_{i}}{k_{i}^{\text {out }}}
$$

where $x_{i}$ is the out-degree of the node $i$. Thus, $b_{i}$ is given by $b_{i}=\left\{\begin{array}{ll}0 & \text { if } \text { in }- \text { degree } \\ 1 & \text { otherwise }\end{array}\right.$.

The closeness centrality $C_{i}$ calculates the proximity among determinants and identifies which is the one closest to all others in the network [23,34-36]. $C_{i}$ is defined by

$$
C_{i}=\sum_{i j} \frac{n}{g_{i j}}
$$

where $n$ represents the total number of the shortest paths (the shortest self-avoiding route that runs from one determinant to another along with the connectivity [34]) between the determinant $i$ and $j$, and $g_{i j}$ is each elementary shortest path or the distance between the determinants $i$ and $j$. The betweenness centrality $B_{i}$ measures how a determinant is favorably located to connect other determinants of the network $[24,33,34,37]$. Assuming that $g_{s t}$ is the total number of shortest paths from $s$ to $t$ then $n_{s t}^{i}$ is the number of shortest paths from the determinants $s$ to $t$. Simply 
$n_{s t}^{i}=\left\{\begin{array}{l}1 \text { if there is a relationship between } s \text { and } t \\ 0 \quad \text { otherwise }\end{array}\right.$.

The computation of $B_{i}$ is given by

$B_{i}=\sum_{s t} \frac{n_{s t}^{i}}{g_{s t}}$

All the analyses were run using $\mathrm{R}[38]$.

All participants involved in this study were informed and signed their consent for the recording and the use of the meetings' materials for scientific purposes.

\section{Results}

\section{A system model of malaria transmission and persistence}

The transmission and persistence of malaria in Accra are portrayed in a CLD of the complex system model, entailing 56 interactions among 45 determinants. This model shows three submodels triggered each by a reinforcing loop, i.e., (i) the urbanization-related transmission and acquired resistance of Anopheles to insecticides (green), (ii) the human's infection-prone behavior (red), and (iii) the healthcare efficiency and Plasmodium resistance (blue) (Fig1). Urbanization-related transmission and resistance of Anopheles to insecticides

The deficient city planning and the planning enforcement, inadequate housing conditions, and limited waste and sewage infrastructure lead to the proliferation of Anopheles breeding sites, which is worsened by the excavation of wells for urban and peri-urban agriculture and rainfall. Besides, a temperature range between 26 and $33^{\circ} \mathrm{C}$ in Accra, contributes to the increase in the reproductive rate of Anopheles, their absolute numbers, and finally their survival, augmenting the risk of infection.

Furthermore, the preventive use of insecticides in households, agricultural sites, and hospitals leaves residues all contribute to the development of insecticide resistance in local mosquito populations. Thus, in the reinforcing loop one (R1, Fig1), we observe that the transmission of 
malaria depends not only on the environmental factors, such as temperature and rainfall but also on the lack of regulations to prevent and control the proliferation of mosquito breeding sites. These effects are exacerbated by the widespread use of insecticides. Hence, this reinforcing loop portrayed the environment as a pathway of both, the infection and the development of resistance of mosquitoes to insecticides (Fig. 1).

\section{Humans infection-prone behavior}

At the individual and household levels, ideally, the awareness of malaria risk leads to the reduction of nighttime activities, as well as the use of protective/preventive measures against mosquito bites, such as the use of insect-proof mesh for doors and windows and insecticidetreated bed-nets (ITNs). The more these measures are accepted and used, the lower the infection will be. In addition, human migration increases the number of infected cases by importation. More infected people in Accra imply a greater number of mosquitoes becoming infected that will subsequently transmit the pathogens to new hosts. This reinforcing loop two (R2, Fig 1) highlights the importance of individual and household decisions, and how a changing behavior can prevent the transmission and its persistence by, for instance, reducing the nighttime activities and using protective measures like ITNs. (Fig 1.).

\section{Healthcare efficiency and Plasmodium resistance}

Malaria carriers can be asymptomatic, and as Ghana's healthcare system is often unable to detect them, they frequently remain untreated and thus keep spreading the disease. Knowledge of malaria symptomatology and a sufficient household income lead to more visits to healthcare facilities. If healthcare workers are well trained, adhere to prescription protocols, and patients comply with the prescribed treatment, the reinforcing loop three (R3, Fig1) will operate, and trust in the health system will grow. If not, the use of inadequate medication will increase, 
alongside the resistance of the Plasmodium parasite to preventive and curative drugs. Besides, the free availability of heavily subsidized drug treatments augments self-medication and indirectly enhances drug-resistance development in Plasmodium. This loop reveals that a good healthcare system requires to be well endowed logistically and in terms of personnel.

Substituting such a healthcare system with highly accessible low-priced drugs can increase the prevalence of resistant strains of Plasmodium. Moreover, this loop revealed an unintended pathway of malaria treatment policies [30], which, although well-intentioned, can be counterproductive. Relatedly, symptomatic patients, unsatisfied with allopathic treatments and drug resistance, may opt for alternative medicines, despite its often uncertain outcomes.

[Insert Fig 1 here]

Fig. 1: Causal loop diagram depicting the complexity in malaria transmission and persistence in Accra (Ghana). In green the urbanization-related transmission sub-model, in red the human's infection-prone behavior of malaria sub-model, and the healthcare efficiency and Plasmodium resistance sub-model (blue)

\section{Network analysis of the CLD}

\section{Properties of the network}

The network representing the CLD displayed a structure of small-world, meaning that all determinants are not interconnected but are anyhow reachable by a small number of steps [39]. It shows an average path of 6.309 , meaning that each determinant can reach any other in average through 6.309 paths. Still, it has a low density (0.028), presenting only $2.8 \%$ of possible edges in a completely interconnected network, and suggesting that a change in a determinant will have only a limited impact on the whole system. This indicates that despite its apparent complexity, 
there is a small connection path among determinants, allowing the information to spread rapidly $[24,40]$. Furthermore, a modularity of 0.619 implies a structural clustering among determinants, indicating that acting on the determinants of the highest betweenness will have a spillover effect on the whole system [24]. In other words, an effective way to impulse a change in the system is to induce a change in the mediator.

Network metrics

The CLD has a scale-free distribution, meaning that its in-degree and out-degree metrics showed a heavy-tailed distribution with values ranging from 0 to 5 (Suppl. Fig. 2). Few nodes show 0 out-degree, indicating that most of the determinants influence other determinants. This configuration describes well real-world networks and suggests a high resilience of the system [41] (Table $1 \&$ Suppl. Fig. 2). Thus, beyond the mediator of the system, the other leverage points also needed to be strategically adjusted to efficiently finetune the system.

The network centrality metrics revealed that malaria positive cases, was the determinant of higher centrality, either impacting or been impacted by seven determinants. Also, the number of breeding sites was impacted by five other determinants, $\left(k^{i n}=5\right)$ namely: 1. more rainfall, 2. the hygiene and sanitation of householders' compound, 3. the adequate housing construction, 4. a convenient waste and sewage management, and 5. the wells excavation. Conversely, the householders' awareness and decision-making on malaria infection risk was the main cause of transmission and persistence, as it impacts five other determinants $\left(k^{\text {out }}=5\right)$ namely 1 . the hygiene and sanitation of household compound, 2. the use of ITN, 3. the frequency and duration of nighttime activities, 4. the use of door and windows mesh, and 5. self-medication.

Predictably, the determinant of highest betweenness that connects most clusters of determinants is the malaria positive cases $(B=328)$; and the one with the highest Page rank, and, thus, the 
most influential is the malaria positive cases $(x=0.086)$. Also, the determinant with the greatest closeness centrality, i.e., with the shortest distance to all others is drug prescription $(C=0.381)($ Table 1$)$.

Interestingly, determinants participating directly in the infection process, such as the number of breeding sites, the malaria positive cases, or not, such as the drug prescription and householders' awareness and decision-making on malaria infection risk are also important leverage points (influential points in the system where a small change in these determinants can induce a big change in the whole system [42]) that can affect the system.

[Insert Table 1 here]

\section{Discussion}

\section{System model of malaria transmission and persistence in Accra}

We found that the most central determinants also standing for the leverage points of the system, aside from the environmental-related ones, are those resulting from the citizens' awareness of malaria infection risks, and household income, and derived empowerment. These findings corroborate earlier studies that showed that poor-income households are more vulnerable to the disease, by facing a double burden: Malaria hotspots in Accra are in economically deprived communities, where malaria infection risk is additionally fueled by the economic needs, leading to community members to take up jobs that increase their exposure [43]; and, poor-income households spend relatively more of their earnings on the treatment of malaria than the higherincome ones [44].

Our results also stress the importance of an efficient healthcare system, a structural issue in most countries of the Global South. We found particularly relevant the trust in the healthcare system, which can be reinforced through training and supervision of health workers on malaria diagnosis 
and treatment-related protocols, and the adherence of the health workers to it. Supporting this, a system dynamics simulation on policies for improving neonatal health in Uganda demonstrated that the workload of healthcare workers affects the development of trust of patients, especially when it leads to long waiting times for attention [45]. Likewise, a study in England and Wales revealed that the reduction of waiting-time built the trust of patients and enhanced healthcare efficiency [46]. On the other hand, the non-adherence to the prescription and treatment protocols by health workers can lead communities to underestimate malaria infection and increase its effects, which augments the distrust in the health system.

Relatedly, we found that the subsidy on anti-malaria drugs instead of promoting a more holistic public health policy contributes to patients' self-medication [47], highlighting the sometimes counterproductive effects of a sole focus of public health programs on biomedical policies as this ignores the complexity of malaria transmission [48]. Previous research showed that patients' selfmedication leads to arbitrary dosage and posology of anti-malaria drugs, and tends to exacerbate the symptoms and augments the morbidity and mortality of malaria [49]. Such situations are worse in poor-income households, where people often self-medicate with inadequate or counterfeit drugs, and/or inappropriate dosages and posologies at the onset of malaria symptoms [47].

Humans can be infected with P. falciparum and be symptomatic or asymptomatic. The latter is most often undiagnosed, among others, because its testing is costly [50]. That is the reason why many public health systems tend to neglect them, thereby contributing to the persistence of malaria [51]. Also, imported cases by human migration amplify the number of cases in cities and at times can initiate the resurgence of malaria in locations where it had been previously under control $[52,53]$. 
Our findings also suggest that deficient urban sanitation and poor urban planning increase the number of mosquito breeding sites. About $10 \%$ of Anopheles mosquitoes breeding sites in Accra are situated around construction sites [12]. Similar observations were made in Nigeria and Tanzania, where clogged gutters and sewage channels are playing similar roles [55]. Thus, the ecology- and behavior-related adaptations of the mosquitoes delay control and make such efforts less effective, thereby contributing to the persistence of malaria in cities [56].

\section{Emergent properties of malaria transmission}

The small-world and scale-free properties that feature our CLD indicate that the network is resilient and the identified leverage points could help to set more adequate policy recommendations.

The functional analysis of the network allowed to identify the determinants of the more central standing for potential intervention points such as causal, impacted, closest, mediator, and influential, and derive from them key leverage points. Thus, we found that i) the malaria positive cases was both, the most influential and the greatest mediator; and ii) the number of breeding sites had the larger effects. These findings align with a recent review of malaria determinants for sub-Saharan Africa [57] that highlights the surge of infection as an intricate interplay between mosquitoes, humans, and their environments. Furthermore, i) the drug prescription was the closest determinant, and ii) the householders' awareness and decision-making on malaria infection risk the most important cause. This indicates that malaria transmission and persistence rely heavily on human behavior, which opens opportunities for more targeted policy action. The CLD was able to disclose the interactions among malaria determinants and also permitted to track the causal links among them that preserve transmission and the feedback loops that 
reinforce certain sets of determinants [58], which permits signaling emerging properties of the system post the NA [59].

\section{Conclusions}

The proposed CLD contributed to illustrate the complexity of malaria transmission and

persistence in our case study, Accra, Ghana. It showed that beyond the mere biological processes and the physical environment, the behavior of people play a key role in malaria transmission and persistence. The CLD embodies three major loops that trigger and maintain transmissions in urban environments. Furthermore, the NA enabled the detection of emergent properties of the system and the identification of the key levering determinants. Besides, the topology disclosed by the CLD revealed that all leverage points need to be accounted for strategic policy development. Hence, major efforts toward preventing malaria transmission are needed, and on that, the key priorities should be: to reduce malaria persistence by reducing mosquito density, for instance, through the regular drainage of gutters or treating breeding sites with larvicides; and reducing infections by increasing the awareness of city dwellers on malaria literacy, for instance, through regular campaigns in deprived communities, both, on the field and social media. Ongoing measures, like, protecting windows and doors with mosquito-proof netting and the use of ITNs, should be intensified. Besides, an improvement of the healthcare system through regular training of the healthcare workers in malaria can enhance trust in the healthcare system and limit the risk of patients' non-compliance to malaria-drugs prescription.

\section{Authors disclaimers}

The above-mentioned findings and conclusions of this study are those of the authors and do not represent the official position of the Center for Development Research (ZEF). Besides, the findings 
have not been influenced by the Ministry of Culture and Science of the Federal State of North Rhine-Westphalia who supported this study.

\section{Authors contributions}

All authors fulfill the contribution requirements as per the International Committee of Medical Journal Editors' role of authors and contributors' guidelines. MKS: conception, data analysis, software, drafts; DCC: conception and drafts; HEZT \& CB: drafts and supervision.

\section{Acknowledgments:}

The authors want to thank Caroline O. Buckee, Keith Smith, Florent Noulèkoun, Timo Falkenberg, and Daniel Larremore for the pieces of advice provided alongside the improvement of this manuscript. The authors are, indeed grateful for the active participation of the experts in our workshops and would like to thank them as well as the supporting team.

\section{Funding:}

This work was financially supported by the Ministry of Culture and Science of North RhineWestphalia.

\section{Conflicts of interest:}

The co-authors of this manuscript have any competing interests to declare

\section{Ethical approval}

The study was approved by the ethics committees of the Center for Development Research, the University of Bonn (Germany), and the Institute of Statistical, Social and Economic Research, University of Ghana.

\section{Availability of data and materials}

All relevant data are provided in the manuscript or available from published materials as cited. 


\section{References}

1. WHO. Malaria [Internet]. 2020 [cited 2020 Jun 16]. Available from:

https://www.who.int/news-room/fact-sheets/detail/malaria

2. WHO/UNICEF. WHO | Africa Malaria Report 2003. World Heal. Organ. Nations Child. Fund. World Health Organization; 2015.

3. Mwabu GM, Orem JN, Kirigia JM, Okorosobo T, Okorosobo F, Mwabu G. Economic Burden of Malaria in six Countries of Africa [Internet]. Eur. J. Bus. Manag. www.iiste.org ISSN. Online; 2011. Available from: https://www.researchgate.net/publication/232725098

4. World Health Organization. World malaria report 2017 [Internet]. 2017. Available from: http://apps.who.int/iris/bitstream/10665/259492/1/9789241565523-eng.pdf

5. Donnelly MJ, McCall PJ, Lengeler C, Bates I, D’Alessandro U, Barnish G, et al. Malaria and urbanization in sub-Saharan Africa. Malar. J. 2005.

6. Owusu G, Yankson PWK. Urbanization in Ghana. Econ Ghana Sixty Years after Indep [Internet]. Oxford University Press; 2017 [cited 2019 Sep 29]. p. 207-22. Available from: http://www.oxfordscholarship.com/view/10.1093/acprof:oso/9780198753438.001.0001/acprof9780198753438-chapter-13

7. Cabannes Y. The impact of participatory budgeting on basic services: municipal practices and evidence from the field. Environ Urban. 2015;27:257-84.

8. Elsey H, Agyepong I, Huque R, Quayyem Z, Baral S, Ebenso B, et al. Rethinking health systems in the context of urbanisation: Challenges from four rapidly urbanising low-income and middle-income countries. BMJ Glob Heal. BMJ Publishing Group; 2019;4. 
9. Lattof SR. Health insurance and care-seeking behaviours of female migrants in Accra, Ghana. Health Policy Plan [Internet]. 2018 [cited 2020 Jul 10];33:505-15. Available from: https://academic.oup.com/heapol/article/33/4/505/4862500

10. Keiser J, Utzinger J, Castro MC De, Smith TA, Tanner M, Singer BH. Urbanization in SubSaharan Africa and Implication for Malaria Control [Internet]. Breman JG, Alilio MS, Mills A, editors. Am. J. Trop. Med. Hyg. American Society of Tropical Medicine and Hygiene; 2004. Available from: https://www.ncbi.nlm.nih.gov/books/NBK3775/

11. Kabaria CW, Gilbert M, Noor AM, Snow RW, Linard C. The impact of urbanization and population density on childhood Plasmodium falciparum parasite prevalence rates in Africa. Malar J [Internet]. BioMed Central Ltd.; 2017 [cited 2020 Jun 16];16:49. Available from: http://malariajournal.biomedcentral.com/articles/10.1186/s12936-017-1694-2

12. Mattah PAD, Futagbi G, Amekudzi LK, Mattah MM, De Souza DK, Kartey-Attipoe WD, et al. Diversity in breeding sites and distribution of Anopheles mosquitoes in selected urban areas of southern Ghana. Parasites and Vectors [Internet]. BioMed Central Ltd.; 2017 [cited 2019 Sep 23];10:25. Available from: http://parasitesandvectors.biomedcentral.com/articles/10.1186/s13071-016-1941-3

13. Endo N, Eltahir EAB. Environmental determinants of malaria transmission in African villages. Malar J. BioMed Central Ltd.; 2016;15.

14. Williams HA, Jones $\mathrm{COH}$. A critical review of behavioral issues related to malaria control in sub-Saharan Africa: What contributions have social scientists made? Soc. Sci. Med. 2004. p. 501-23. 
15. Adome RO, Whyte SR, Hardon A. Popular Pills: Community Drug Use in Uganda [Internet]. Het Spinhuis; 1996 [cited 2019 Sep 30]. Available from:

http://books.google.com/books?hl=en\&lr=\&id=S8SetwRrzOsC\&pgis=1

16. Van Der Geest S. Self-care and the informal sale of drugs in South Cameroon. Soc Sci Med. 1987;25:293-305.

17. Mitchell M. Complexity: A Guided Tour. Oxford. Oxford University Press; 2011.

18. Berkes F, Folke C, Colding J. Navigating Social-Ecological Systems: Building Resilience for Complexity and Change. Berkes F, Folke C, Colding J, editors. Biol Conserv. 1st ed. Cambridge University Press; 2004;119:581.

19. Ludwig VB. An Outline of General System Theory. Br J Philos Sci. 1950;1:134-65.

20. Walker B, Carpenter S, Anderies J, Abel N, Cumming G, Janssen M, et al. Resilience management in social-ecological systems: A working hypothesis for a participatory approach. Ecol Soc. Resilience Alliance; 2002;6.

21. Baugh Littlejohns L, Baum F, Lawless A, Freeman T. The value of a causal loop diagram in exploring the complex interplay of factors that influence health promotion in a multisectoral health system in Australia. Heal Res Policy Syst. BioMed Central Ltd.; 2018;16.

22. Borgatti SP, Halgin DS. On network theory. Organ Sci. 2011;

23. Sporns O, Honey CJ, Kötter R. Identification and classification of hubs in brain networks. PLoS One. 2007;2.

24. McGlashan J, Johnstone M, Creighton D, De La Haye K, Allender S. Quantifying a systems 
map: Network analysis of a childhood obesity causal loop diagram. PLoS One. Public Library of Science; 2016;11.

25. Prell C. Social Network Analysis: : History, Theory and Methodology. 1st ed. University of Groningen - RuG, editor. SAGE Publications Ltd; 2011.

26. Wikipedia. Accra [Internet]. 2020 [cited 2020 Jul 12]. Available from: https://en.wikipedia.org/wiki/Accra

27. Awine T, Malm K, Bart-Plange C, Silal SP. Towards malaria control and elimination in Ghana: challenges and decision making tools to guide planning. Glob Health Action [Internet]. 2017 [cited 2018 Feb 26];10:1381471. Available from:

http://www.ncbi.nlm.nih.gov/pubmed/29035160

28. Songsore J. Environmental and Structural Inequalities in Greater Accra [Internet]. J. Int. Inst. 2008. Available from: http://quod.lib/

29. Austin KF. Dependency, Urban Slums, and the Forgotten Plagues. Sociol Perspect [Internet]. 2015 [cited 2019 Nov 19];58:286-310. Available from:

http://journals.sagepub.com/doi/10.1177/0731121414556542

30. Hovmand PS. Community Based System Dynamics [Internet]. New York, NY: Springer New York; 2014 [cited 2018 Apr 28]. Available from: http://link.springer.com/10.1007/978-14614-8763-0

31. Binder T, Vox A, Belyazid S, Ordur Haraldsson $\mathrm{H}^{*}$. Developing system dynamics models from causal loop diagrams. Proc 22nd Int Conf Syst Dyn Soc. Oxford; 2004.

32. Purwanto A, Sušnik J, Suryadi FX, de Fraiture C. Using group model building to develop a 
causal loop mapping of the water-energy-food security nexus in Karawang Regency, Indonesia. J Clean Prod. Elsevier Ltd; 2019;240:118170.

33. Golbeck J. Analyzing networks. Introd to Soc Media Investig. Elsevier; 2015. p. 221-35.

34. Newman M. Networks. Seconde Ed. Oxford, editor. New York: Oxford University Press; 2018.

35. Sabidussi G. The centrality index of a graph. Psychometrika. Springer-Verlag; 1966;31:581603.

36. Bavelas A. Communication Patterns in Task-Oriented Groups. J Acoust Soc Am. $1950 ; 22: 725-30$.

37. Freeman LC. A Set of Measures of Centrality Based on Betweenness. Sociometry. JSTOR; $1977 ; 40: 35-41$.

38. R Core Team. R: A language and environment for statistical computing. Vienna, Australia: R Foundation for Statistical Computing; 2013.

39. Hexmoor H. Computational Network Science: An Algorithmic Approach. Kaufmann M, editor. Comput. Netw. Sci. An Algorithmic Approach. Elsevier Inc.; 2016.

40. Albert R, Barabási AL, ka Albert R, szló Barabá si A-L. Statistical mechanics of complex networks [Internet]. Rev. Mod. Phys. American Physical Society; Jan 30, 2002 p. 47-97. Available from: https://journals.aps.org/rmp/abstract/10.1103/RevModPhys.74.47

41. Hein O, Schwind M, König W. Scale-free networks: The impact of fat tailed degree distribution on diffusion and communication processes. Wirtschaftsinformatik [Internet]. 
Springer; 2006 [cited 2021 Jan 10];48:267-75. Available from:

https://link.springer.com/article/10.1007/s11576-006-0058-2

42. Fischer J, Riechers M. A leverage points perspective on sustainability. People Nat [Internet]. Wiley; 2019 [cited 2021 Jan 15];1:115-20. Available from:

https://onlinelibrary.wiley.com/doi/abs/10.1002/pan3.13

43. Fobil JN, Levers C, Lakes T, Loag W, Kraemer A, May J. Mapping Urban Malaria and Diarrhea Mortality in Accra, Ghana: Evidence of Vulnerabilities and Implications for Urban Health Policy. J Urban Heal [Internet]. Springer US; 2012 [cited 2018 Feb 3];89:977-91. Available from: http://link.springer.com/10.1007/s11524-012-9702-x

44. Chuma JM, Thiede M, Molyneux CS. Rethinking the economic costs of malaria at the household level: Evidence from applying a new analytical framework in rural Kenya. Malar J. $2006 ; 5$.

45. Rwashana Semwanga A, Nakubulwa S, Adam T. Applying a system dynamics modelling approach to explore policy options for improving neonatal health in Uganda. Heal Res Policy Syst. 2016;14.

46. Calnan MW, Sanford E. Public trust in health care: The system or the doctor? Qual. Saf. Heal. Care. 2004. p. 92-7.

47. Winstanley P, Ward S, Snow R, Breckenridge A. Therapy of falciparum malaria in subSaharan Africa: From molecule to policy. Clin. Microbiol. Rev. 2004. p. 612-37.

48. Haynes A, Garvey K, Davidson S, Milat A. What can policy-makers get out of systems thinking? Policy partners' experiences of a systems-focused research collaboration in preventive 
health. Int J Heal Policy Manag [Internet]. Kerman University of Medical Sciences; 2020 [cited 2021 Jan 11];9:65-76. Available from:/pmc/articles/PMC7054651/?report=abstract

49. Awuah RB, Asante PY, Sakyi L, Biney AAEE, Kushitor MK, Agyei F, et al. Factors associated with treatment-seeking for malaria in urban poor communities in Accra, Ghana. Malar J [Internet]. BioMed Central Ltd.; 2018 [cited 2019 Sep 20];17:168. Available from: https://malariajournal.biomedcentral.com/articles/10.1186/s12936-018-2311-8

50. Sturrock HJW, Hsiang MS, Cohen JM, Smith DL, Greenhouse B, Bousema T, et al. Targeting Asymptomatic Malaria Infections: Active Surveillance in Control and Elimination. PLoS Med [Internet]. Public Library of Science; 2013 [cited 2020 Jul 28];10:e1001467. Available from: https://dx.plos.org/10.1371/journal.pmed.1001467

51. Tao D, McGill B, Hamerly T, Kobayashi T, Khare P, Dziedzic A, et al. A saliva-based rapid test to quantify the infectious subclinical malaria parasite reservoir. Sci Transl Med. American Association for the Advancement of Science; 2019;11.

52. Martens P, Hall L. Malaria on the move: Human population movement and malaria transmission. Emerg. Infect. Dis. Centers for Disease Control and Prevention (CDC); 2000. p. $103-9$.

53. Buckee CO, Wesolowski A, Eagle NN, Hansen E, Snow RW. Mobile phones and malaria: modeling human and parasite travel. Travel Med Infect Dis [Internet]. 2013 [cited 2019 Sep 23];11:15-22. Available from: http://www.ncbi.nlm.nih.gov/pubmed/23478045

54. Damien BG, Baxerres C, Apetoh E, Le Hesran JY. Between traditional remedies and pharmaceutical drugs: prevention and treatment of "Palu" in households in Benin, West Africa. 
BMC Public Health. BioMed Central Ltd; 2020;20.

55. Adeleke M, Mafiana C, Idowu A, Adekunle M, Sam-Wabo S. Mosquito larval habitats and public health implications in Abeokuta, Ogun State, Nigeria. Tanzan J Health Res [Internet]. 2008 [cited 2019 Sep 24];10. Available from:

http://www.ajol.info/index.php/thrb/article/view/14348

56. Wilke ABB, Caban-Martinez AJ, Ajelli M, Vasquez C, Petrie W, Beier JC. Mosquito Adaptation to the Extreme Habitats of Urban Construction Sites. Trends Parasitol. Elsevier Ltd; 2019;35:607-14.

57. De Silva PM, Marshall JM. Factors contributing to urban malaria transmission in sub-saharan Africa: A systematic review. J. Trop. Med. 2012.

58. Rickles D, Hawe P, Shiell A. A simple guide to chaos and complexity. J Epidemiol Community Heal [Internet]. 2007 [cited 2020 Mar 17];61:933-7. Available from: www.jech.com 59. Ahn AC, Tewari M, Poon C-S, Phillips RS. The Limits of Reductionism in Medicine: Could Systems Biology Offer an Alternative? PLoS Med [Internet]. Public Library of Science; 2006 [cited 2020 Mar 17];3:e208. Available from: https://dx.plos.org/10.1371/journal.pmed.0030208 


\section{Figures}

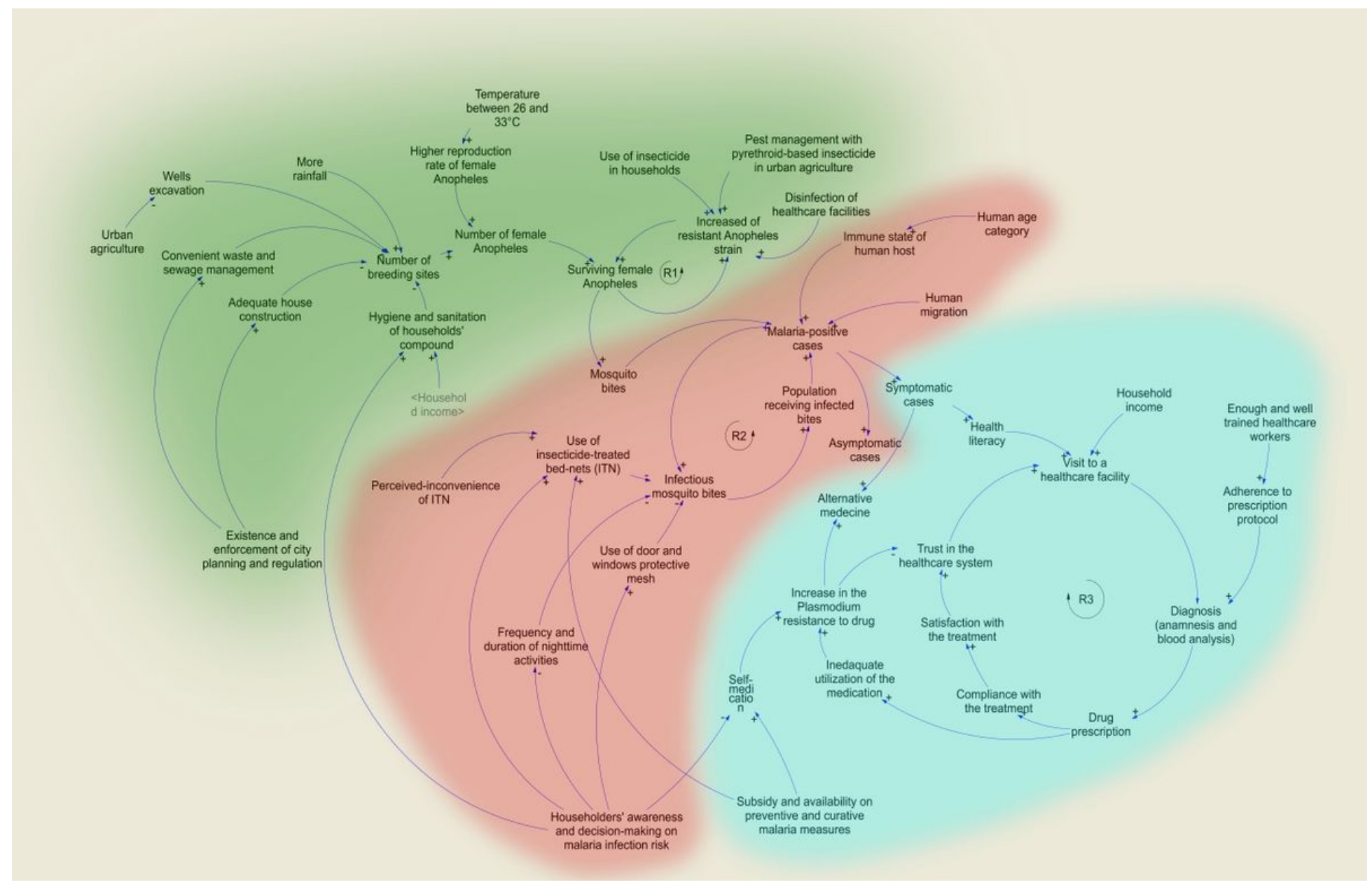

\section{Figure 1}

Causal loop diagram depicting the complexity in malaria transmission and persistence in Accra (Ghana). In green the urbanization-related transmission sub-model, in red the human's infection-prone behavior of malaria sub-model, and the healthcare efficiency and Plasmodium resistance sub-model (blue)

\section{Supplementary Files}

This is a list of supplementary files associated with this preprint. Click to download.

- Supplemental.docx 